\title{
Modified computational design synthesis using simulation- based evaluation and constraint consistency for vehicle powertrain systems
}

Citation for published version (APA):

Wijkniet, J., \& Hofman, T. (2018). Modified computational design synthesis using simulation-based evaluation and constraint consistency for vehicle powertrain systems. IEEE Transactions on Vehicular Technology, 67(9), 8065-8076. [8371654]. https://doi.org/10.1109/TVT.2018.2844024

Document license:

TAVERNE

DOI:

10.1109/TVT.2018.2844024

Document status and date:

Published: 01/09/2018

Document Version:

Publisher's PDF, also known as Version of Record (includes final page, issue and volume numbers)

Please check the document version of this publication:

- A submitted manuscript is the version of the article upon submission and before peer-review. There can be important differences between the submitted version and the official published version of record. People interested in the research are advised to contact the author for the final version of the publication, or visit the $\mathrm{DOI}$ to the publisher's website.

- The final author version and the galley proof are versions of the publication after peer review.

- The final published version features the final layout of the paper including the volume, issue and page numbers.

Link to publication

\footnotetext{
General rights

- You may freely distribute the URL identifying the publication in the public portal. follow below link for the End User Agreement:

www.tue.nl/taverne

\section{Take down policy}

If you believe that this document breaches copyright please contact us at:

openaccess@tue.nl

providing details and we will investigate your claim.
}

Copyright and moral rights for the publications made accessible in the public portal are retained by the authors and/or other copyright owners and it is a condition of accessing publications that users recognise and abide by the legal requirements associated with these rights.

- Users may download and print one copy of any publication from the public portal for the purpose of private study or research.

- You may not further distribute the material or use it for any profit-making activity or commercial gain

If the publication is distributed under the terms of Article 25fa of the Dutch Copyright Act, indicated by the "Taverne" license above, please 


\title{
Modified Computational Design Synthesis Using Simulation-Based Evaluation and Constraint Consistency for Vehicle Powertrain Systems
}

\author{
Jan Wijkniet (1) and Theo Hofman (1)
}

\begin{abstract}
The combinatorial nature of powertrain system design problems challenges system engineers as powertrain components can be interconnected, sized, and controlled in numerous ways. Hence, finding promising candidates considering, e.g., fuel economy, drivability, and complexity requires a systematic approach. A dedicated and novel framework for computational design synthesis is, therefore, presented in this paper. The underlying aim of this framework is to enable full-automated powertrain system optimization over multiple system layers, including topology selection, component sizing, and optimal control, and follows the principles of platform-based design. Based on a library of components, system topologies are automatically generated by solving a constraint satisfaction problem. Meanwhile, constraints originating from customers' requirements, application-specific design rules, and physical laws are respected. Furthermore, the topology generation can be applied to hierarchical decomposed systems on multiple system levels in order to reach the full potential of system design. Using a relevant automotive use case, it is demonstrated that possible topologies for powertrains equipped with a complex continuously variable transmission are automatically synthesized. Next, simulation-based evaluation (automated physical modeling and filtering) of these candidates by this framework results in a set of feasible topologies, satisfying the required functionality and physical constructibility. Along with this topology evaluation phase, a control-actuation scheme and an initial set of feasible component parameters are obtained that are required for full-automated multilayer optimization, which is seen as the next novel future step. The presented framework leads to novel and innovative powertrain and transmission designs with a total amount of 635 feasible transmission topologies based on maximum 13 components, whereas the overall time required to generate and evaluate all initial possible candidates $\left(2.5 \cdot 10^{27}\right)$ is relatively short, i.e., 5.5 and $20 \mathrm{~h}$, respectively.
\end{abstract}

Index Terms-Computational design synthesis, constraint satisfaction programming, constraint consistency, nested graph, topology, powertrain.

$\begin{array}{ll}c & \text { Nomenclature } \\ f^{s, d} & \text { Constraint. } \\ & \text { Static, dynamic function. }\end{array}$

Manuscript received October 17, 2017; revised April 19, 2018; accepted May 29, 2018. Date of publication June 4, 2018; date of current version September 17, 2018. The review of this paper was coordinated by Dr. M. S. Ahmed. (Corresponding author: Theo Hofman.)

J. Wijkniet is with the Punch Powertrain Nederland B.V., Eindhoven 5653 LD, The Netherlands (e-mail: jan.wijkniet@punchpowertrain.com).

T. Hofman is with the Control Systems Technology Group Department of Mechanical Engineering, Eindhoven University of Technology, Eindhoven 5600 MB, The Netherlands (e-mail: t.hofman@tue.nl).

Color versions of one or more of the figures in this paper are available online at http://ieeexplore.ieee.org.

Digital Object Identifier 10.1109/TVT.2018.2844024
Vertex (component).

Equality constraint.

Inequality constraint.

Parallel layout.

Series layout.

Time.

Start, end time.

Control input.

Plant, control variable.

Set of constraints.

Set of edges.

Operational mode.

Set of vertices.

Bijection.

Plant, control objective.

System level.

Sets of constraints.

Sets of edges.

Graph.

Sets of vertices.

Poss., feas. topologies.

Analytical target cascading.

Brake.

Comput. design synthesis.

Clutch.

CP Constraint programming.

CVT Contin. variable transmission.

DR Dual range.

DSM Design structure matrix.

DW Differential set and wheels.

EM Electric machine.

FG Fixed-gear ratio stage.

4WD Four-wheel drive.

GP Gear pair.

ICE Internal combustion engine.

MDM Multi-domain matrix.

MDO Multidisciplinary optimization.

MED Medium drive.

MI Mixed.

NGM Nested graph model.

OD Overdrive.

PGS Planetary gear set.

PHEV Plug-in hybrid electric vehicle.

PS Power spilt.

SR Single range

SS Shaft-to-shaft. 


$\begin{array}{ll}\text { TM } & \text { Transmission. } \\ \text { UD } & \text { underdrive. } \\ \text { VAR } & \text { variator. } \\ \text { VE } & \text { vehicle type. } \\ \text { VN } & \text { virtual node. }\end{array}$

\section{Superscripts}

$\begin{array}{ll}a & \text { application specific. } \\ c & \text { control. } \\ c & \text { cost (only with } c \text { ). } \\ d & \text { dynamic. } \\ f & \text { functional. } \\ k & \text { type of constraint. } \\ p & \text { plant. } \\ p & \text { physical (only with } c \text { ) } \\ s & \text { static. } \\ i, \ldots, q & \text { index variables. } \\ \text { Subscripts } \\ \tau & \text { component type. } \\ \iota & \text { component instance. }\end{array}$

\section{INTRODUCTION}

$\mathbf{P}$ OWERTRAIN system design can be considered as a complex problem due to its multi-disciplinary and combinatorial character; and, as a representative case study for a large class of complex mechatronic system design problems. For optimal and competitive design, three optimization levels should be distinguished: (1) discrete topology (system layout), (2) component technology selection and parametrization, and (3) control design [1]. Optimization with respect to the component and control design has exhaustively been investigated in the past and is considered to be well-established [2]. However, by considering only these two levels, the system layout is (mostly) pre-defined, hence, the design space is very much restricted. Moreover, the modification of the powertrain topology design can improve the system performance significantly, sometimes even more than the component and control design optimization alone [3]. Nonetheless, discrete topology design is considered to be a complicated task, since the number, type and connectivity of components can be specified in various ways.

A generic, commonly-used approach in design synthesis is the platform-based design paradigm [4]-[6]. A platform is defined as a set of components that can be connected to each other to form an embodiment. Further, each of these components are specified in a library and characterized in terms of the number of instances, number of connection points, parameter domain and the functionality it can support [6]. Mapping of the system functionality to a physical design or topology is the main objective of the platform-based design paradigm. Functionality is defined as what the system is supposed to do, whereas a topology represents the way a system does what it is supposed to do [4]. The design process is not fully top-down or bottom-up, but rather a meet-in-the-middle process. The middle is where functionality meets the topology, yielding a feasible design [4], [5], [7]. Using this approach, cyber-physical systems such as, e.g., aircraft electric power systems [6] and hybrid electric vehicles [5], [8] have been successfully synthesized. In order to structure the sequential representation (1), generation (2), evaluation (3) and guidance (4) design steps for complex dynamical systems

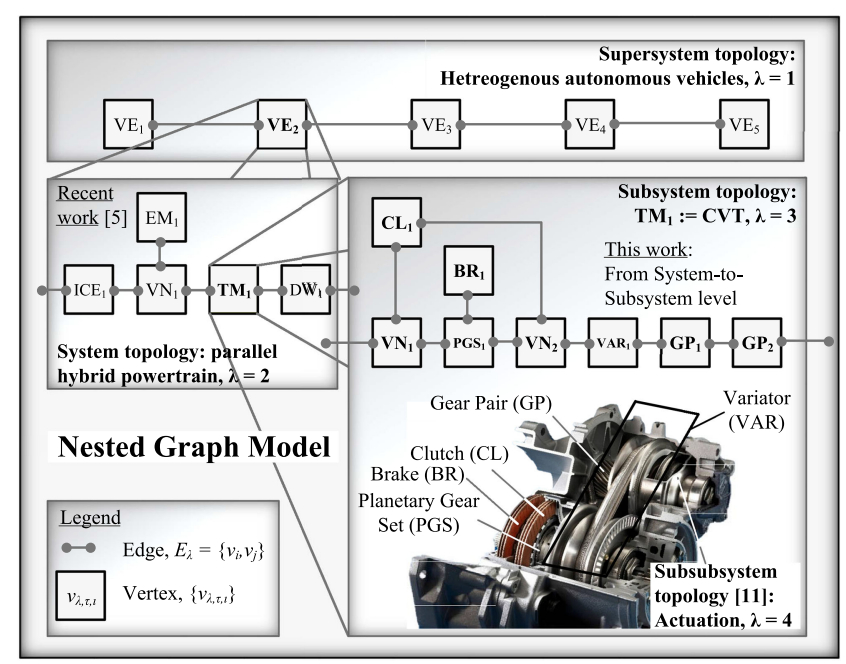

Fig. 1. Nested graph model representing discrete dynamic system topologies from higher system to lower system level. Cutaway of a conventional continuously variable transmission (CVT) shows some physical component elements at subsystem $(\lambda=3)$ and subsubsystem level $(\lambda=4)$ (Cf. Fig. 2 and Table I).

[9], [10], the constraint programming (CP) technique is formulated within a modified generic computational design synthesis (CDS) framework. This novel framework extends the topology generation problem, as proposed by [5] and enables automateddiscrete-topology-design synthesis from higher to lower levels in sequential steps using:

- a nested graph model (NGM) to represent the hierarchical dynamic system topology decomposition in step (1);

- two additional type of subset constraints: physical and application specific constraints in step (2);

- a simulation-based evaluation approach using automated physical modeling and filtering in step (3); and, finally,

- a cyclic model to achieve constraint consistency between the feasible topologies of different sequential system levels in step (4).

In [5], the topology generation was limited to the system level (Cf. Fig. 1) and the above mentioned contributions are realized by actually investigating the implementation of the method from [5] to lower system levels (Cf. Fig. 2). In [11], the topology synthesis for new electro-mechanical or -hydraulical actuation system is explained and complementary to the work described here. Furthermore, this general framework aims to enable automated optimization on the three system optimization layers (topology, component, control) in near future. The 'optimization' that will be performed in this paper is used as an evaluation step to filter out infeasible topologies more early in the design process reducing computation time. In [12] and [13], we present a novel method, which allows to make the automated transitional step from the multi-level 'architectural models' presented in this paper to 'analytical analysis models' for arbitrary electrical and hybrid powertrains (incl. all-feasible discrete operational mode schemes). These parameterized dynamical models can be used for fuel, emissions or battery energy minimization subject to an optimal control strategy (e.g., Pontryagin's minimum principle) [14]), whereby the components (power source, transmission elements) can be automatically optimized. Applications of this 


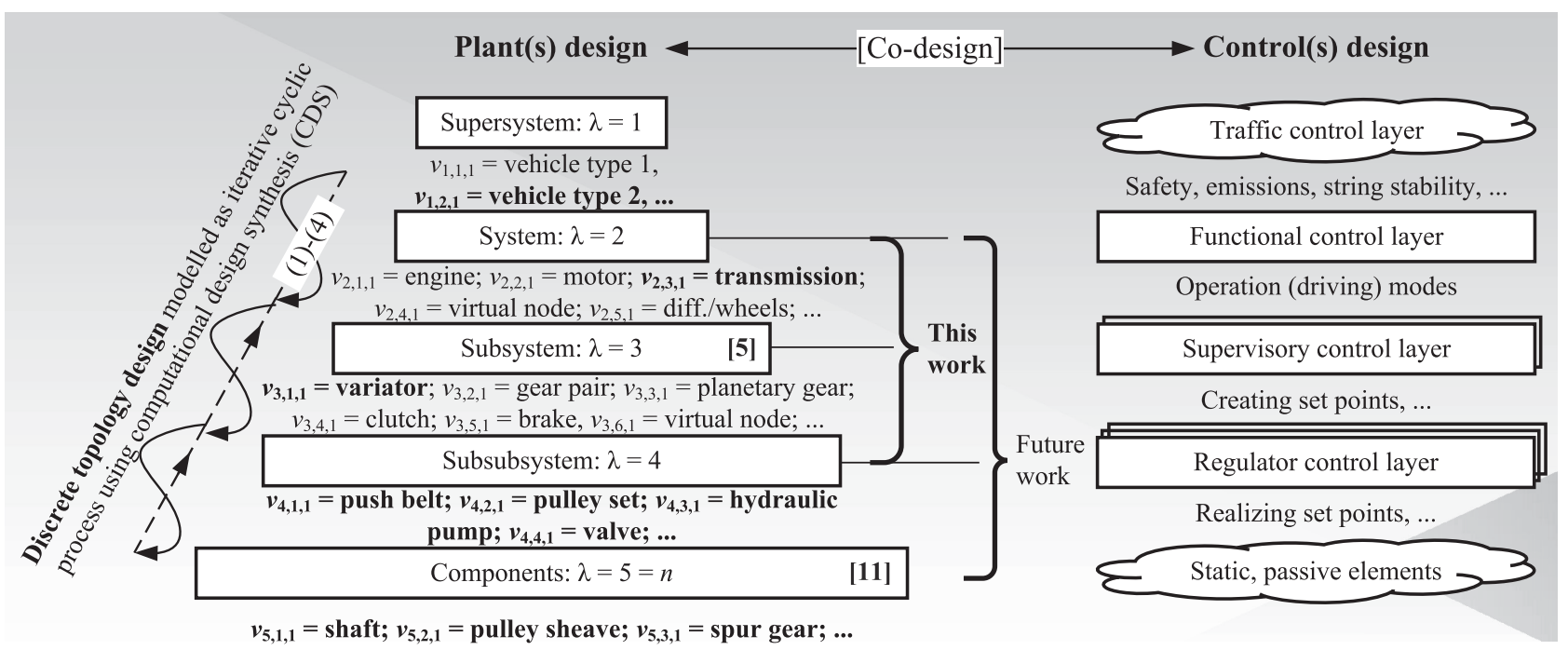

Fig. 2. Hierarchical system level decomposition including examples of automotive powertrain (sub)systems and components, where the powertrain type is defined as the system level. The multidisciplinary optimization (MDO) framework (co-design) is extended with computational design synthesis (CDS) (Cf. Fig. 3) for discrete topologies using nested graph modeling (NGM) (Cf. Fig. 1). Moreover, in an iterative cyclic process, additional consistency constraints as input to step (1) are defined assuring that, e.g., the operation modes at the higher levels are realized with feasible lower-level system topologies. These constraints are formulated and implemented at the end of each CDS loop (guidance, step (4), Fig. 3).

framework, besides the automotive domain, may include any other dynamical (industrial) application, e.g., in the healthcare, semiconductor equipment or mechatronic domains, that contains a high level of complexity. The strength of the proposed novel framework is its flexibility, high level of detail, modularity and robustness to different applications and domains. The article is organized as follows: Section II proposes the modified and improved framework that integrates automated discrete topology design synthesis on multiple system levels and is demonstrated by means of a case study in Section III. For this case study, the topology generation of electric-hybrid powertrain systems equipped with a front-transverse push-belt CVT is performed. Section IV concludes with findings and recommendations for future research.

\section{CDS Framework Modification AND REFINEMENT}

Below, more details concerning the computational design synthesis (CDS) framework modifications and refinements are provided in correspondence with the discussed sequential design steps (1)-(4) from the previous section.

\section{A. Nested Graph Model Representing a Hierarchical Dynamic System Topology Decomposition in Step (1)}

A complex dynamic engineering system can hierarchically be decomposed from 'supersystem level' to 'component level' and each system level $\lambda$ contains a certain set of subsystems or components, denoted as $V_{\lambda}=\left\{v_{\lambda, \tau, \iota} \mid \tau, \iota \in \mathbb{N}_{1}, \lambda \in \Lambda \subset \mathbb{N}_{1}\right\}$. The subscripts $\tau$ and $\iota$ indicate, respectively, the system element type (e.g., engine, CVT, etc.) and the number of instances that the same system element type occurs in the topology at a system level $\lambda$. Further, note that a higher system element (as being part of a topology), $v_{\lambda, \tau, \iota}$ uses a particular set (e.g., if not all elements are used for being functional) of elements from a lower level $\left\{v_{\lambda+1, \tau, \iota}\right\}$ that belongs to a subset (in the particular case) of the set of lower level elements, $v_{\lambda, \tau, L}=\left\{\left\{v_{\lambda+1, \tau, L}\right\} \mid\left\{v_{\lambda+1, \tau, L}\right\} \subseteq V_{\lambda+1}\right\}$, which can be represented as an undirected, unweighed, connected and finite graph $\mathcal{G}_{\lambda}($.$) ; and, is expressed as an order pair with,$ $T_{\lambda, \tau, \iota}=\mathcal{G}_{\lambda}\left(V_{\lambda+1}, E_{\lambda+1}\right) \subseteq V_{\lambda+1} \times E_{\lambda+1}$ with the edges $E_{\lambda+1}$ defining the connection of the subsystem elements or vertices $V_{\lambda+1}$. The edges $E_{\lambda+1}$ can be expressed as a set of twoelement subsets of the set of subsystem elements $V_{\lambda+1}$ with $E_{\lambda+1} \subseteq\left[V_{\lambda+1}\right]^{2}$.

Therefore, in correspondence with the decomposition from higher to lower system levels, a higher system level topology (graph model) $\mathcal{G}_{\lambda}($.$) consists of a set of multiple lower system$ level topologies (subgraph models), $\mathcal{G}_{\lambda+1}($.$) with V_{\lambda+1} \subseteq V_{\lambda}$ and $E_{\lambda+1} \subseteq E_{\lambda}$. For multiple system levels this will result in a nested graph model (NGM) of finite size as shown in Fig. 1 with a limit on the cardinality of the topology sets [15] with $\mathbb{V}=\bigcup_{\lambda=1}^{n} V_{\lambda}$ and $\mathbb{E}=\bigcup_{\lambda=1}^{n} E_{\lambda}$ for all the possible system elements and edges. Hierarchical decomposition (using clustering of (sub)graphs or advanced partitioning) in step (1) can be done, e.g., based on: the functional analysis (design structural matrix (DSM) methods [16]); or, the existence of associated features, such as the spatial partition or physical boundaries around subsystems. In [17], multi-domain matrices (MDM) are created based upon mapping the functions-to-functions with a functional DSM, functions-to-components with a design mapping matrix (DMD) and components-to-components with a component DSM. By mathematical manipulation, i.e., the differences $\triangle \mathrm{MDM}$ and summation $\sum \mathrm{MDM}$ of the resulting MDMs for several powertrain architectures, results in insights in (i) the architectural differences and (ii) enables to generate (component and architectural) design rules, creates typology definition (architecture type, drive configuration, transmission type, and E-motor placement), and a generic DMD for all investigated 
architectures, respectively. However, the automated topology generation and system optimization is still seen as one of the major challenges. Therefore, in this work, we focus in particular on this challenge and see opportunities in the future to automate the process of constraint generation as well using MDM methods.

\section{B. Dynamical System Topology Modeling and Optimization}

Two types of components can be defined: (i) input and output components defining the system boundary; and, (ii) physical parts composing the system [18]. Additionally, abstractions can be made with respect to the system functionality to confine the design space. Let us assume that every dynamical physical system (vertex) comprises two modelling concepts describing the related static (volume, mass, etc.) and dynamic behavior (angular speed, torque, state-of-charge, etc.) properties. Depending on the designer's problem setup, the functions mapping the control $x^{c}$ (power split, gear shift command, clutch command, etc.) and plant design decisions $x^{p}$ (swept volume, ratio coverage, etc.) to these properties, denoted as $f^{d}($.$) and f^{s}($.$) , respectively, can$ also be used to calculate the control $\phi^{c}$ (.) (fuel usage, emissions, etc.) or plant performances $\phi^{p}$ (.) (total-cost-of-ownership, etc.). Each subsystem, as part of a feasible system topology, has also inherently static and dynamical properties, however, assume that it is possible to link each system property individually to a particular subsystem that matches this property the most. This can easily be done by looking at which of the subsystems are activelycontrolled or not. In the latter case, the subsystem is denoted as being a static or passive subsystem. After this process the vertices (as subsystems) are transformed into plant and control design variables using the same notation for the ease of reading: $\left\{v_{\lambda+1}^{p}\right\}:=x^{p}$ and $\left\{v_{\lambda+1}^{c}\right\}:=x^{c}$. These newly defined variables are used to map the static properties with $f^{s}:\left\{v_{\lambda+1}^{p}\right\} \longmapsto v_{\lambda}^{p}$ or the dynamic properties with $f^{d}:\left\{v_{\lambda+1}^{c}\right\} \longmapsto v_{\lambda}^{c}$, respectively, to the static and dynamic properties of the preceding system level. For example, looking at the topologies in Fig. 1, we see that $\left\{v_{3}\right\}=\left\{v_{3}^{c}\right\} \cup\left\{v_{3}^{p}\right\}=\left\{\mathrm{CL}_{1}, \mathrm{BR}_{1}, \mathrm{VAR}_{1}\right\} \cup$ $\left\{\mathrm{PGS}_{1}, \mathrm{GP}_{1}, \mathrm{GP}_{2}, \overline{\mathrm{VAR}}_{1}, \mathrm{VAR}_{1}\right\}$. Here, e.g., not only the ratio values for the planetary gear sets PGS, gear pairs GP's, yet also the over- $\left({ }^{-}\right)$and under-drive speed ratio (.) of the CVT can be seen as plant optimization parameters. The clutch $\mathrm{CL}_{1}$, brake $\mathrm{BR}_{1}$ and variator $\mathrm{VAR}_{1}$ are components of which their controlled states (open/close, or speed ratio) are determined by the optimized reference signals (as targets) from a higher system level, i.e., the overall transmission ratio $f^{d}:\left\{\mathrm{CL}_{1}, \mathrm{BR}_{1}, \mathrm{VAR}_{1}\right\} \mapsto \mathrm{TM}_{1}$ with $\mathrm{TM}_{1} \in\left\{v_{2}^{c}\right\}$. In addition, the overall transmission ratio, is determined by the plant parameters from the lower system level as well, i.e., $f^{s}:\left\{\mathrm{PGS}_{1}, \mathrm{GP}_{1}, \mathrm{GP}_{2}, \overline{\mathrm{VAR}}_{1}, \underline{\mathrm{VAR}}_{1}\right\} \mapsto\left\{\overline{\mathrm{TM}}_{1}, \mathrm{TM}_{1}\right\}$ with $\left\{\overline{\mathrm{TM}}_{1}, \mathrm{TM}_{1}\right\} \subset\left\{v_{2}^{p}\right\}$. Using these formulations, the dynamic behavior of a system over a finite time horizon $\left[t_{0}, t_{f}\right]$ is captured by the state-variable trajectories $\xi_{\lambda}^{c}(t):\left[t_{0}, t_{f}\right] \longmapsto \Omega_{\lambda}^{c} \subseteq \mathbb{R}^{n}$ and the static properties with the plant design variables $x^{p} \longmapsto$ $\Omega_{\lambda}^{p} \subseteq \mathbb{R}^{m}$. If we assume, an active-controlled plant system design, with $u_{\lambda}^{c}(t)$ as the control inputs, aiming at minimizing a certain local (plant and/or control) system target $\phi_{\lambda}^{p, c}($.) as design specification, then the fully-integrated plant and control design (co-design) optimization problem becomes [3]:

$$
\min _{x_{\lambda}^{c}(t), x_{\lambda}^{p}} \phi_{\lambda}^{p, c}(\underbrace{\xi_{\lambda}^{c}(t), u_{\lambda}^{c}(t)}_{x_{\lambda}^{c}(t)}, x_{\lambda}^{p}, t)
$$

subject to: $g_{\lambda}: f_{\lambda}^{s, d}\left(\xi_{\lambda}^{c}(t), u_{\lambda}^{c}(t), x_{\lambda}^{p}\right) \leq 0$

$$
h_{\lambda}: \dot{\xi}_{\lambda}^{c}(t)-f_{\lambda}^{d}\left(\xi_{\lambda}^{c}(t), u_{\lambda}^{c}(t), x_{\lambda}^{p}, t\right)=0
$$

The (in-)equality constraints at each system level $\lambda$ are represented by $g_{\lambda}$ and $h_{\lambda}$, respectively. In most cases, the plant design $f^{s}($.$) does not depend on the control variables \left(\xi_{\lambda}^{c}, u_{\lambda}^{c}\right)$, yet the dynamic properties $f^{d}($.$) do depend on the plant design x^{p}$ allowing a nested design approach. A classical object-based system decomposition is depicted on the left-side in Fig. 2. Moreover, for controlled-dynamical systems, the same hierarchical decomposition type can be made related to the control design, as depicted on the right-side in Fig. 2, from the 'functional', 'supervisory' to the 'regulator control layer'. The 'traffic control layer' may regulate the heterogeneous platooning vehicle topologies digitally coupled via (wireless) communication (using decentralized or distributed control) in a road transportation environment maximizing traffic safety, cost savings and efficiency. Elements at component level are assumed to be 'static' or 'passive dynamic' elements that require no closed-loop control. The original system design problem is partitioned over two domains: plant (topology, component technology) and control design resulting in a 'co-design' framework. The nested graph model (NGM) representation enables the multidisciplinary optimization (MDO) framework to be extended with discrete-topology synthesis using computation design synthesis (CDS) based on constraint programming $(\mathrm{CP})$ for dynamical systems as will be discussed next.

\section{Constraint Satisfaction Programming Extended Using Physical and Application Specific Constraints in Step (2)}

The topology synthesis and search process for feasible designs in step (2) can be formalized as a constraint programming (CP) problem and can be defined as:

$$
\begin{aligned}
\text { find all : } & \mathbb{T}_{\lambda}^{\mathrm{F}} \subseteq \mathbb{T}_{\lambda}^{\mathrm{P}} \\
\text { subject to : } \quad & c_{\lambda, m}^{k} \in C_{\lambda} \subseteq \mathbb{C} \\
\forall & m \in\{1, \ldots, q\} \subseteq \mathbb{N}_{1} ; k \in\{f, c, p, a\} ; \\
& \lambda \in\{1, \ldots, n\} \subseteq \mathbb{N}_{1} ; \mathbb{C}=\bigcup_{\lambda=1}^{n-1} C_{\lambda}
\end{aligned}
$$

where $\lambda$ denotes again the system level and $k$ the constraint type with a specific number $m$. The parameter $q^{k}$ represents the total amount of constraints for a particular constraint type $k$. Furthermore, a distinction is made between the set of possible $(\mathrm{P})$ and feasible topologies $(\mathrm{F}), \mathbb{T}_{\lambda}^{\mathrm{P}}$ and $\mathbb{T}_{\lambda}^{\mathrm{F}}$, respectively. In this context, feasible refers in a qualitative manner to: physically possible or constructible; do not contain redundant components or subsystems; contains at least the required system operation modes to be functional; and, finally, meets the performance requirements satisfying the different type of constraints: functionality $(k=f)$, redundancy $(k=r)$, physical $(k=p)$, application 
specific $(k=a)$, denoted as $c_{\lambda, m}^{k}$. The set $\mathbb{C}$ represents the complete set of constraints for the problem. The amount of elementary components or the cardinality of $V_{\lambda}$, denoted as $\left|V_{\lambda}\right|$ may vary with each level $\lambda$, since $v_{\lambda, \tau, \iota} \in V_{\lambda}$ for the double tuple $(\tau, \iota)=\{\{\tau\},\{\tau, \iota\}\} \subseteq \mathbb{N}_{1} \times \mathbb{N}_{1}$. The same holds for the cardinality of $C_{\lambda}$ and, therefore, the total number of constraints for each given system level $\lambda$ varies with $\left|C_{\lambda}\right|$. The $\mathrm{CP}$ is solved using the Prolog software [19].

\section{Simulation-Based Evaluation Using Automated Physical Modeling and Filtering in Step (3)}

The evaluation step consists of four intermediate steps from: (3a) screening; (3b) mode searching; (3c) performance evaluation; towards (3d) classification (typology definition) of feasible topologies. Using the topology description from step (2), the topology uniqueness (by solving the graph isomorphism problem of labeled graphs) is evaluated in (3a). Accordingly, the synthesized and unique topologies are automatically transformed into dynamic simulation models in using the Simscape software within the Matlab Simulink environment [20] in step (3b). Using the constructed parameterized dynamic models, a set of valid plant design parameters and a control-actuation scheme or dynamic-operation modes based on a power-flow path analysis are automatically specified for each topology [12] and [13]. Finally, the topology performances in terms of reaching certain design targets and satisfying constraints are evaluated in step (3c). In case of minimization of fuel, emissions or batteryenergy usage, an optimal control strategy can be utilized. Other performance design models, e.g., but not limited to: acceleration, power reserve, etc., can be taken into design consideration as well. The topologies are categorized in step (3d) for analysis by the designer. Note for the steps (3a)-(3d), script files (.m) programmed in Matlab are used.

\section{E. Constraint Consistency by Implementation of the Cyclic CDS Model in Step (4)}

The result of step (3) is a reduced set of feasible topologies. These topology sets can input a MDO framework (e.g., analytical target cascading (ATC) [21]), which optimizes this set of topologies with respect to predefined objective and constraint functions. Moreover, the system engineer or the CDS algorithm (when automated) can select, e.g., based on these optimization results and with additional requirements that originate from the market (if we, e.g., consider powertrain system level design, $\lambda=2$ ), a particular topology from this feasible topology set in order to generate feasible topologies at a lower-system level $(\lambda=3)$. By doing so, proper constraint handling between these two system levels is a necessity for guaranteeing functionality of lower-system level topologies. For example, generating a powertrain consisting of two electric machines and a combustion engine requires a transmission design that couples these three inputs (hence forcing these three components to be always present on transmission level $(\lambda=3)$ to the output, being the wheels. Therefore, the CDS process described by the four sequential design steps can be modelled as an iterative cycle process from higher to lower system levels assuring constraint consistency by formulating new constraints in the guidance step (4)

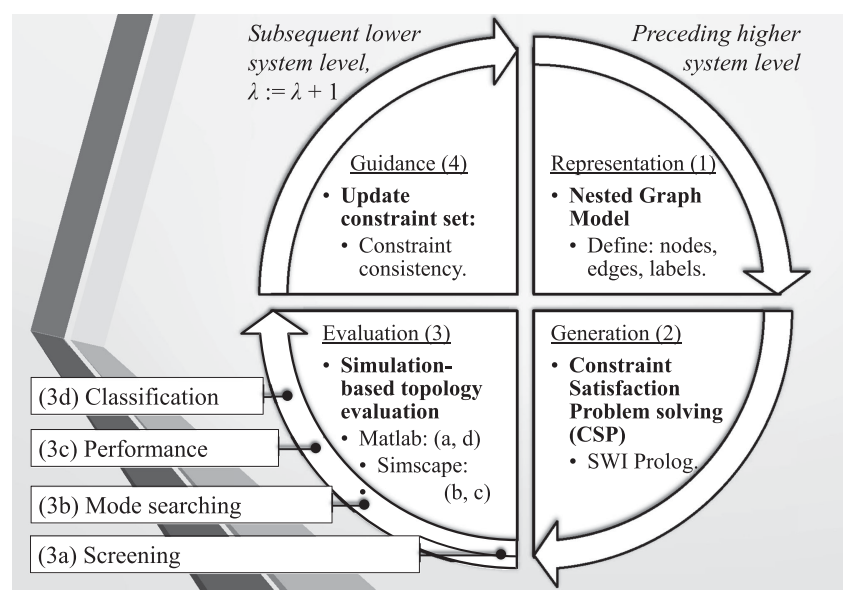

Fig. 3. A generic computational design synthesis (CDS) framework that is modified for automated-discrete-topology selection from the system to component level in sequential steps using a cyclic model. Novel attributes to this process of feasible topology search are 'simulation-based evaluation' (step (3)) and 'constraint consistency' (step (4)) realization by matching the requirements from higher system level to lower level topology design constraints.

as input to step (1). This process is summarized and shown in the Figs. 2 and 3, respectively.

\section{Method Validation: Powertrain Topology SYNTHESIS CASE STUDY}

The effectiveness of the proposed modified CDS framework is demonstrated in this section using an automotive powertrain. An automotive powertrain tries to match via a transmission the required power at the wheels to the available engine and/or electric machine(s) power in an optimal way. For this study, as transmission technology, a front-transverse push-belt CVT application, coupled to an internal combustion engine (ICE) and/or electric machine(s) (EM), is selected. A conventional CVT, typically, consists of a finite set of elementary mechanical and hydraulic components and a cutaway is depicted in Fig. 1. These components can be combined to each other in a finite, yet large number of ways. A CVT is a promising transmission type in terms of efficiency and comfort [22], [23] and the number of CVTs sold worldwide is increasing. Recently, more complex topologies are introduced [24], enlarging the strong need to synthesize novel and innovative CVT topologies in a structured way. Further, it is assumed that the input shaft of a transmission always rotates in clockwise direction, viewed from the engine side. In addition, the transmission is assumed to be located on the left-hand side of a vehicle, viewed from the rear. Hence, for forward driving, the output shaft of the transmission is compelled to rotate in clockwise direction as well. Furthermore, the variator in this case study is assumed to be a conventional push-belt type. The powertrain topology synthesis $(\lambda=2)$ is included to explain the cyclic model to achieve architectural constraint consistency between different sequential system levels $(\lambda=2 \rightarrow \lambda=3)$. Design synthesis of an actuation subsystem (hydraulic, electromechanical, etc., $\lambda=4$ ), being part of a CVT, is excluded from this case study. However, without loss of generality, it can be performed in the same way as elaborated for the CVT system 
TABLE I POWERTRAIN SyStem PLATFORM: $v_{1,2,1}^{p, c}=\left\{v_{2,1 \ldots 5,1 \ldots 3}^{p, c}\right\} \subseteq V_{2}^{p} \cup V_{2}^{c}$

\begin{tabular}{lllccc}
\hline $\begin{array}{l}\text { Component } \\
\text { type, } \tau\end{array}$ & $\begin{array}{l}\text { Design variable } \\
\text { type, } v_{\lambda, \tau, \iota}^{p, c}\end{array}$ & $\begin{array}{l}\text { Layout } \\
\text { color }\end{array}$ & $\begin{array}{c}\text { Number of } \\
\text { Instances, } \sum \iota\end{array}$ & $\begin{array}{c}\text { Degree, } \\
\operatorname{deg}\left(v_{\lambda, \tau, \iota}^{p, c}\right)\end{array}$ \\
\hline $1:$ & $\mathrm{ICE}_{1}$ & $v_{2,1,1}^{p}:$ input & $\square$ & 1 & 1 \\
$2:$ & $\mathrm{EM}_{1 \ldots 2}$ & $v_{2,2,1 \ldots 3}^{p, c}$ & $\square$ & 2 & 1 \\
$3:$ & $\mathrm{TM}_{1 \ldots 2}$ & $v_{2,1,1 \ldots 2}^{p, c}$ & $\square$ & 2 & 2 \\
$4:$ & $\mathrm{VN}_{1 \ldots 4}$ & $v_{2,4,1 \ldots 4}^{p}, \ldots$ & $\square$ & 3 \\
$5:$ & $\mathrm{DW}_{1 \ldots 2}$ & $v_{2,5,1 \ldots 2}^{p}:$ output & $\square$ & 2 & 1 \\
\hline \multicolumn{5}{l}{$p=$ plant; $c=$ control; $\mathrm{n} / \mathrm{a}=$ not applicable }
\end{tabular}

TABLE II CVT SUBSYSTEM PLATFORM: $v_{2,3,1 \ldots 3}^{p, c}=\left\{v_{3,1 \ldots 6,1 \ldots 3}^{p, c} \subseteq V_{3}^{p} \cup V_{3}^{c}\right.$

\begin{tabular}{lllccc}
\hline $\begin{array}{l}\text { Component } \\
\text { type, } \tau\end{array}$ & $\begin{array}{l}\text { Design variable } \\
\text { type, } v_{\lambda, \tau, \iota}^{p, c}\end{array}$ & $\begin{array}{l}\text { Layout } \\
\text { type/color }\end{array}$ & $\begin{array}{c}\text { Number of } \\
\text { Instances, } \sum \iota\end{array}$ & $\begin{array}{c}\text { Degree, } \\
\mathbf{d e g}\left(v_{\lambda, \tau, \iota}^{p, c}\right)\end{array}$ \\
\hline $1:$ & $\mathrm{VAR}_{1}$ & $v_{3,1,1}^{p, c}$ & $\mathrm{p} \square$ & 1 & 2 \\
$2:$ & $\mathrm{GP}_{1 \ldots 3}$ & $v_{3,2,1 \ldots 3}^{p}$ & $\mathrm{p} \square$ & 3 & 2 \\
$3:$ & $\mathrm{PGS}_{1}$ & $v_{3,3,1}^{p}$ & $\mathrm{~s} \square$ & 1 & 3 \\
$4:$ & $\mathrm{CL}_{1 \ldots 2}$ & $v_{3,4,1 \ldots 2}^{c}$ & $\mathrm{~s} \square$ & 2 & 2 \\
$5:$ & $\mathrm{BR}_{1}$ & $v_{3,5,1}^{c}$ & $\mathrm{~s} \square$ & 1 & 1 \\
$6:$ & $\mathrm{VN}_{1 \ldots 3} \square$ & $v_{3,6,1 \ldots 3}^{p}$ & $\mathrm{~s} \square$ & 3 & 3 \\
\hline \multicolumn{5}{r}{} \\
\end{tabular}

below and is seen also for future research (Cf. Fig. 2). First, the powertrain topology synthesis is discussed below.

\section{A. Powertrain Topology Synthesis Problem}

Here, we define the goal of this design synthesis step to generate powertrain topologies $(\lambda=2)$ following certain 'market requirements' as an relevant example case study. A transmission (TM) is required in order to match the energy load and source(s) specifications. The vehicle loads on either or both the front and rear-axle drive systems are described by a differential component with driven wheels, denoted as DW. Further, we assumed that the powertrain energy sources can be an ICE and/or up to a maximum of two electric machines (EMs) combined. It should be noted that at this level, the number of inputs and outputs of the transmission (TM) are coupled by means of a virtual node, $\mathrm{VN}$. In this case, the VN has no mechanical meaning others than just assembling all the available inputs (EMs, ICE) or outputs (DWs). These nodes could be represented by components from lower-system levels $(\lambda=3)$, e.g., PGS, or GP, etc. The number and type of these inputs and outputs, significantly, influences the design of the TM, i.e., design synthesis problem at $\lambda=3$.

1) Formalizing Functional and Cost Constraints From Market Requirements: The market requirements (top-down) define the constraints and the library of components at system and subsystem level that will form a feasible system platform in the generation step (2) (bottom-up). Here, we used engineering knowledge to define the library of components at both system levels. These library of components are listed in Tables I and II, respectively. Example relevant market requirement are: (i) the powertrain is of the (plug-in) hybrid-electric vehicle (PHEV) type; (ii) is of the class four-wheel drive (4WD); (iii) contains a mechanical-CVT connected (in)directly to the ICE; (iv) the TM (i.e., CVT) enables mechanically (via the ICE) forward and reverse driving; (v) provides full-EV mode; (vi) contains basic hybrid functionalities such as EM charging and assist. The first four market requirements immediately impose the following constraints: (i) $\sum(\mathrm{EM}, \mathrm{ICE}) \geq 2$ at $\lambda=2$; (ii) $\sum \mathrm{DW}_{i}=2$ with $v_{2,5,1 \ldots 2}$ equals a $\mathrm{DW}_{i}$ at $\lambda=2$; (iii) $v_{2,3,1}$ equals a CVT at $\lambda=2$ and $v_{3,1,1}$ equals a variator at $\lambda=3$, respectively; (iv) the topologies at $\lambda=3$, which do not comply with these functional modes will be filter out of the set of possible topologies in step (3) of the CDS process (Cf. Section III-D). Other example functional or cost constraints are: no TM (in)directly between the DWs are allowed, i.e., only between a DW and a source (ICE, EM); and, a maximum of two EMs may be connected to the same transmission. In Section III-B, more details on the library of components for the CVT, the representation, etc., and all other subsequent steps in the CDS process will be provided. First, using the powertrain design case study and market requirements as described above, below an example will be discussed of an actual build system that can be generated with the constraints provided in this paper. For completeness, all functional and cost constraints implemented to generate feasible topologies at powertrain system level are listed in the Table III.

2) Powertrain Topology Representation: An example of the generated powertrain topologies (output step (2)) represents an actual build hybrid system equipped with a mechanical-CVT for the front wheels [25]; and, with an electric drive system for the rear wheels[26]. This powertrain in series production provides a 4WD performance and can switch between modes to operate in full-EV and parallel-hybrid mode. The key components of this multi-mode hybrid on powertrain level $(\lambda=2)$ are the front transmission $\left(\mathrm{TM}_{1}:=\mathrm{CVT}\right)$ that is connected to the engine (ICE) and e-machine $\left(\mathrm{EM}_{1}\right)$. In Fig. 4, the generated solution is represented by a graph diagram (left) at a high level of abstraction and pictures of the actual-build systems (right). Moreover, given the set of components at system level, the amount of possible topologies is $\left|\mathbb{T}_{2}^{\mathrm{P}}\right|: 2^{(11+11 \cdot 10 / 2)}=2^{66} \approx 7.4 \cdot 10^{19}$. Using the imposed functional (11) and cost constraints (12), more concurrent topologies (equipped with a CVT) can be generated and the amount of (initial) set of feasible topology generated (16) are shown in theFig. 6(a). Using this set of generated topologies, they can be classified into several types (2WD, $4 \mathrm{WD}$, etc.), and the diagram in Fig. 6(a) at the top-right summarizes the results. To connect the higher level to lower topologie levels, we assumed here, to focus on a particular topology: 4WD with $\mathrm{TM}_{1}:=\mathrm{CVT}$. It should be noted that we observed that generating the nested topologies from a higher to a lower-system level the amount and type of constraints increase rapidly (from 23 to 174 ) as will be discussed in more details in following sections.

\section{B. Topology CVT Representation, Step (1)}

A stick and a graph diagram of a conventional CVT (Cf. Fig. 1) is depicted inFig. 6(b). The labels of these vertices are specified in Table II. At $\lambda=3$, the input system is the ICE or $v_{2,1,1}^{p}$ and/or EMs, $v_{2,1, \ldots 3}^{p}$ and the output system is the combined differential and driven wheels (DWs) at front- and rearaxles, $v_{2,5,1 \ldots 2}^{p}$. At subsystem level $\lambda=3$, a virtual vertex $\left(v_{3,6, l}^{p}\right)$ can represent a shaft, flange, synchronizer, or any other physical concentric connection element that couples or splits torque (usually, a component from $\lambda=4$ ) [5]. A clutch $\left(v_{3,4,1 \ldots 2}^{c}\right)$ connected to the transmission housing is represented by a brake $\left(v_{3,5,1}^{c}\right)$, 
TABLE III

FunCTIONAL \& COST CONSTRAINTS $c_{2,1 \ldots 11}^{f}, c_{2,1 \ldots 12}^{c} \in C_{2}$ FOR THE POWERTRAIN SYSTEM PLATFORM: $v_{1,2,1}^{p, c}=\left\{v_{2,1 \ldots 5,1 \ldots 3}^{p, c}\right\} \subseteq V_{2}^{p} \cup V_{2}^{c}$ $\sum_{\tau, j}\left(v_{2,4, q}, v_{2, \tau, r}\right)=3 \cdot v_{2,4, q}$

Linking the component together

$c_{2,2}^{f} \quad$ No self-loops allowed

$c_{2,3}^{f} \quad$ No self-loops allowed: 3 VNs

$c_{2,4}^{f} \quad$ No self-loops allowed: 4 VNs

$\sum_{\tau, j}\left(v_{2, \tau, q}, v_{2, \tau, q}\right)=0$

$\left(v_{2,4, q}, v_{2,4, r}\right)+\left(v_{2,4, r}, v_{2,4, s}\right)+$

$\left(v_{2,4, q}, v_{2,4, s}\right)<3$

$\left(v_{2,4, q}, v_{2,4, r}\right)+\left(v_{2,4, q}, v_{2,4, s}\right)+$

$\left(v_{2,4, s}, v_{2,4, t}\right)+\left(v_{2,4, r}, v_{2,4, t}\right)<4$

$\left(v_{2,3, m}, v_{2,4, q}\right)+\left(v_{2,4, q}, v_{2,4, r}\right)+$

$\left(v_{2,4, r}, v_{2,3, m}\right)<3$

$\left(v_{2,3,1}, v_{2,3,2}\right)=0$

$\left(v_{2,3, m}, v_{2,4, q}\right)=1 \rightarrow\left(v_{2,4, q}, v_{2,3, n}\right)=0$

$\left(v_{2,3, m}, v_{2,4, q}\right)+\left(v_{2,4, q}, v_{2,4, r}\right)=2 \rightarrow$

$\left(v_{2,4, r}, v_{2,3, n}\right)=0$

$\left(v_{2,3, m}, v_{2,4, q}\right)+\left(v_{2,4, q}, v_{2,4, r}\right)+$

$\left(v_{2,4, r}, v_{2,4, s}\right)=2 \rightarrow\left(v_{2,4, s}, v_{2,3, n}\right)=0$

$\left(v_{2,2, i}, v_{2,2, j}\right)=0$

$\left(v_{2,2, i}, v_{2,3, m}\right)=1 \rightarrow\left(v_{2,3, m}, v_{2,2, j}\right)=0$

$\left(v_{2,2, i}, v_{2,5, m}\right)=0$

$\left(v_{2,1,1}, v_{2,5, m}\right)=0$

$\left(v_{2,1,1}, v_{2,4, q}\right)=1 \rightarrow\left(v_{2,4, q}, v_{2,5, m}\right)=0$

$\left(v_{2,1,1}, v_{2,4, q}\right)+\left(v_{2,4, q}, v_{2,4, r}\right)=2 \rightarrow$

$\left(v_{2,4, r}, v_{2,5, m}\right)=0$

$\left(v_{2,1,1}, v_{2,4, q}\right)+\left(v_{2,4, q}, v_{2,4, r}\right)+$

$\left(v_{2,4, r}, v_{2,4, s}\right)=3 \rightarrow\left(v_{2,4, s}, v_{2,5, m}\right)=0$

$\left(v_{2,1,1}, v_{2,4, q}\right)+\left(v_{2,4, q}, v_{2,4, r}\right)+$

$\left(v_{2,4, r}, v_{2,4, s}\right)+\left(v_{2, q, s}, v_{2, q, t}\right)=4 \rightarrow$

$\left(v_{2,4, t}, v_{2,5, m}\right)=0$

$\left(v_{2,2, i}, v_{2,4, q}\right)=1 \rightarrow\left(v_{2,4, q}, v_{2,5, m}\right)=0$

$\left(v_{2,2, i}, v_{2,4, q}\right)+\left(v_{2,4, q}, v_{2,4, r}\right)=2 \rightarrow$

$\left(v_{2,4, r}, v_{2,5, m}\right)=0$

$\left(v_{2,2, i}, v_{2,4, q}\right)+\left(v_{2,4, q}, v_{2,4, r}\right)+$

$\left(v_{2,4, r}, v_{2,4, s}\right)=3 \rightarrow\left(v_{2,4, s}, v_{2,5, m}\right)=0$

$\left(v_{2,2, i}, v_{2,4, q}\right)+\left(v_{2,4, q}, v_{2,4, r}\right)+$

$\left(v_{2,4, r}, v_{2,4, s}\right)+\left(v_{2,4, s}, v_{2,4, t}\right)=4 \rightarrow$

$\left(v_{2,4, t}, v_{2,5, m}\right)=0$

$\left(v_{2,2, i}, v_{2,4, q}\right)+\left(v_{2,2, j}, v_{2,4, q}\right)=2 \rightarrow$

$\left(v_{2,2, k}, v_{2,4, q}\right)=0$

$\sum\left(\sum_{i=1}^{3}\left(v_{2,2, i}\right)+v_{2,1,1}\right)>0$ connected to a VN

$c_{2,12}^{c}$ Min. one (energy) source (EM, ICE) is present

$\tau \in\{1, . ., 5\} ; m, n \in\{1,2\} ; i, j, k, p \in\{1,2,3\} ; q, r, s, t \in\{1,2,3,4\}$

since connecting any other component to the housing would result in a redundant system. Moreover, the variator $\left(v_{3,1,1}^{p, c}\right)$, the clutch $\left(v_{3,4,1 \ldots 2}^{c}\right)$ and the brake $\left(v_{3,5,1}^{c}\right)$ are seen as dynamically controlled subsystems with specified 'control' design variables, denoted as $c$. Their continuous and discrete values influence the route of the power flow. The remaining component types for the CVT are defined as 'plant' design variables $p$, since they contribute to the system functionality or driving modes and their values influence the kinematical and kinetic characteristics. In case of the VAR, this component comprises both characteristics: speed ratio and over/underdrive values as controlled states and plant parameters, respectively. Furthermore, the component types can be referred to as a 'series' (s) or a 'parallel' (p) connecting device. Series means that the component can be used

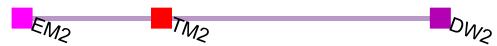

rear wheels [27]

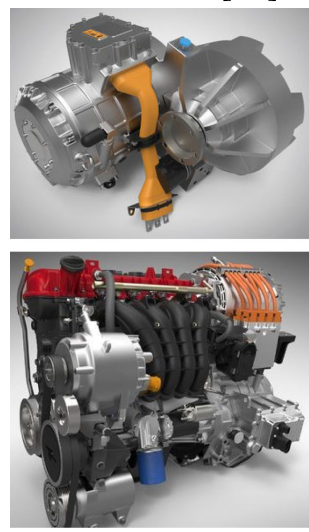

front wheels [26]

Fig. 4. A graph representation of an hybrid electric vehicle powertrain that is currently in production. This powertrain topology consist of components from Table I, building a hybrid front axle $\left(\mathrm{EM}_{1}, \mathrm{ICE}_{1}, \mathrm{TM}_{1}:=\mathrm{CVT}, \mathrm{DW}_{1}\right)$ and an electric rear axle $\left(\mathrm{EM}_{2}, \mathrm{TM}_{2}, \mathrm{DW}_{2}\right)$.

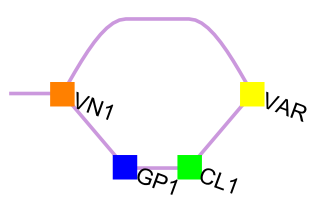

(a)

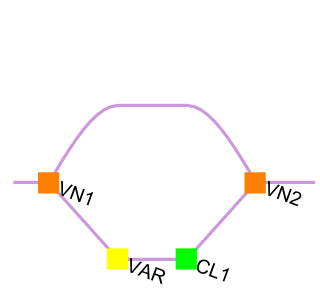

(c)

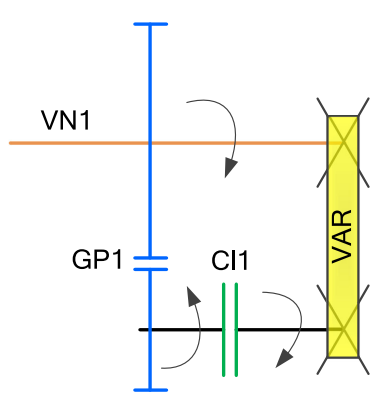

(b)

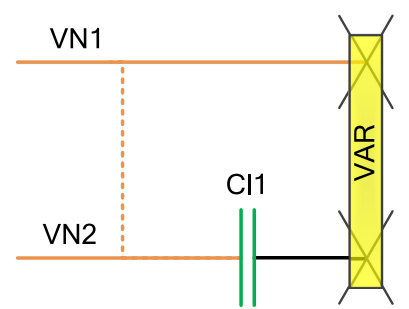

(d)
Fig. 5. Step (2), graph and stick diagram of a counter examples of: plant redundancy and physical constraint in one subsystem (a-b); and, a physical constraint: co-axiallity issue of shafts VN1 and VN2 (c-d).

to connected in a series arrangement of component elements, whereas parallel type refers to an element that allow connecting components in parallel (or, e.g., two or multiple ranges of elements in series connected) (Cf. Fig. 6(b)). In our case, this component type identification can be interpreted as an additional functional constraint, eliminating topologies that could prevent (unwanted) closed-loops between components (e.g., GP-VN$\mathrm{CL}$, the clutch output can not be connected to the input of the GP). The 'degree' of a component, $\operatorname{deg}\left(v_{\lambda, \tau, \iota}^{p, c}\right)$ refers to the number of edges $\left(E_{\lambda}\right)$ that it contains. For this powertrain design case study, following inclusion-exclusion principle with $\left|\bigcup_{\lambda=3}^{4} V_{\lambda}\right|$ a total of $13\left(=\left|v_{2,1 \ldots 3,1}\right|+\left|v_{3,1 \ldots 6,1 \ldots 3}\right|-\right.$ 

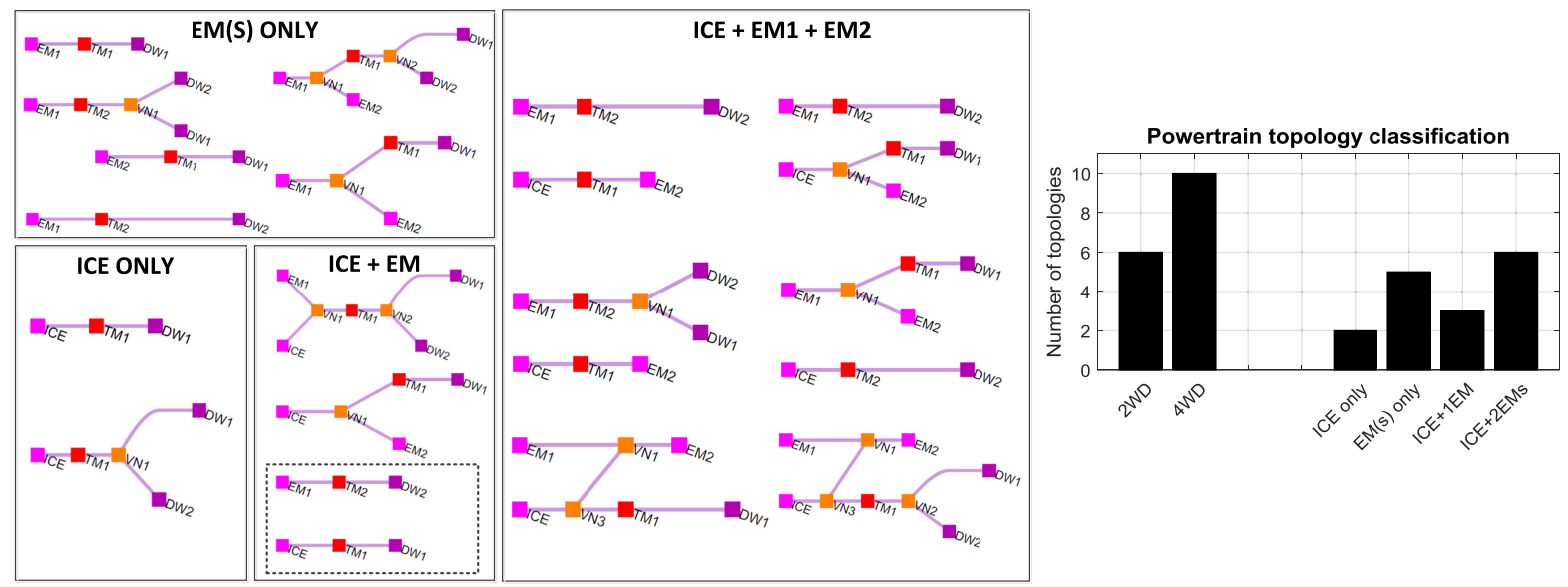

(a)

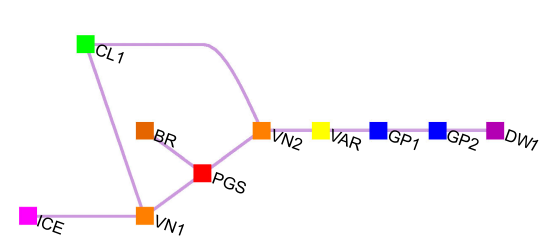

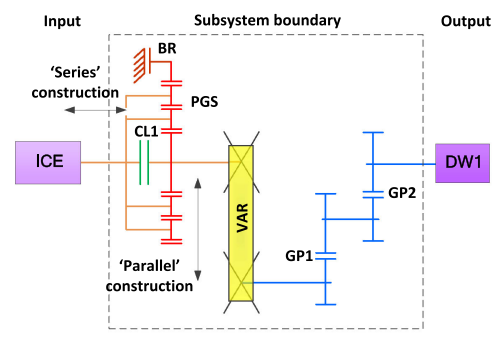

(b)

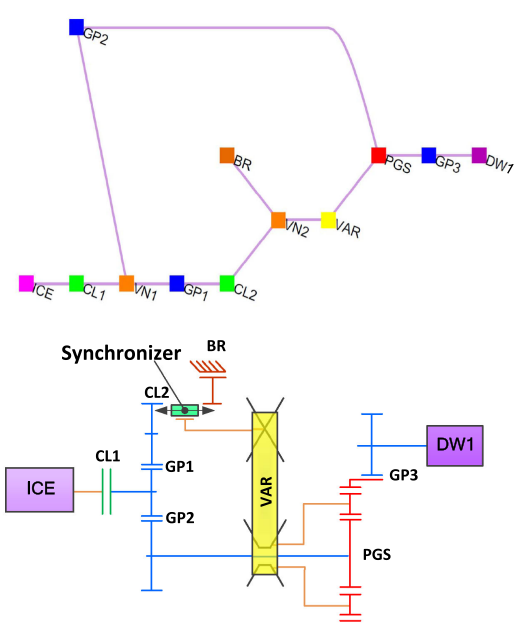

(c)
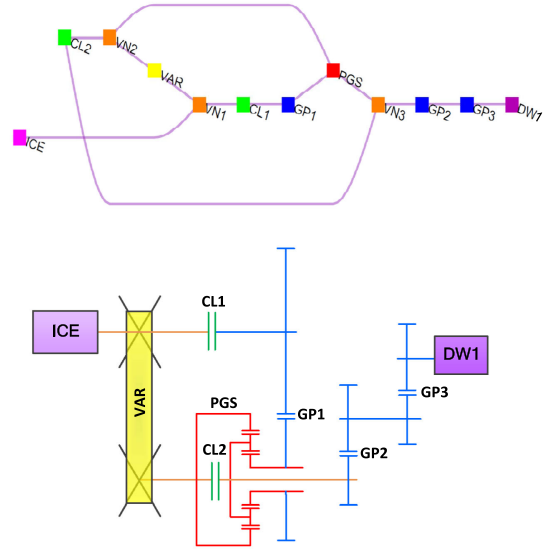

(d)

Fig. 6. Step (3d) at $\lambda=2$ (top) and, accordingly, Step (2) at $\lambda=3$ (bottom), with some examples of automated generated CVT topologies: feasible computational synthesized designs that comprise three different CVT classes: shaft-to-shaft; powersplit; and, mixed. The component labels are specified in Table II. (a) Step (3d): Classification of the generated feasible conventional, all-electric and hybrid-electric powertrain topologies at $\lambda=2$ (from steps (1)-(2)). Here, TM $1:=\mathrm{CVT}$ system, resulting in the generated topologies for $\mathrm{TM}_{1}$ (and the $4 \mathrm{WD}$ topology in the dashed box) as, e.g., shown in (b)-(d) below at $\lambda=3$. (b) single range (SR) shaft-to-shaft (SS). (c) single range (SR) powersplit (PS). (d) mixed (MI): combined SR PS and SR SS.

$\left.\left|v_{2,1 \ldots 3,1} \cap v_{3,1 \ldots 6,1 \ldots 3}\right|=3+11-1\right)$ components constitute the library (and not 14, since $v_{2,2,1}=\left\{v_{3,1 \ldots 6,1 \ldots 3}\right\}$ ). The arrangement of a third degree planetary gear set (PGS) $\left(v_{3,3,1}\right)$ is essential for its functionality. Two options are possible to include that phenomena in a $\mathrm{CP}$ algorithm: extend the component identity with an edge number; or, perform a global search over $(3 !)^{\iota}$ possible arrangements during the mode searching process. The second option is preferred due to its lower computational effort and is further elaborated next.

\section{Automated CVT Topology Generation, Step (2)}

The variables of the constraint programming $(\mathrm{CP})$ are the components $V_{\lambda}$, as listed in the Table II and their interconnections defined by $E_{\lambda}$. A binary domain is defined for each variable, stating that a variable is either present or absent. The amount of possible topologies can be again calculated with $\left|\mathbb{T}_{3}^{\mathrm{P}}\right|: 2^{(13+13 \cdot 12 / 2)}=2^{91} \approx 2.5 \cdot 10^{27}$. The CP is solved using the Prolog software [19]. Thereby, four categories of constraints are defined for this mechanical system, resulting in: 22 functionality; 48 redundancy; 79 physical; 25 application specific constraints; and, hence, in a total of 174 constraints programmed in about 4600 lines of code in Prolog; which, are discussed below in a qualitative manner including some specific examples.

1) Functionality Constraints: The functionality constraints are defined on two levels: subsystem $(\lambda=3)$ and system level $(\lambda=2)$. On subsystem level, the graph should be consistent, i.e., all edges should be connected. In addition, the cardinality should be respected. For example, if a third degree subsystem is present in a system, then three edges containing that subsystem must exist. Finally, the formation of self loops must be prevented. A group of subsubsystems must be ensured to function adequately on system level. For example, a brake (BR) may only be present if a PGS is present and may only be connected to a VN or PGS directly. On system level $(\lambda=2)$, the system is compelled to have boundaries, i.e., input and output, and should be able to meet the performance requirements, as defined by the designer (Cf. Section III-A). 
2) Redundancy Constraints: The redundancy constraints can be grouped into two categories: plant and control-type related. Plant redundancy constraints are related to reduced or eliminated functionality of the mechanical subsystems. For example, connecting two ports of a PGS to a particular subsystem eliminates one degree of freedom of the PGS. The same holds for the mechanical loops through which no mechanical power is transferred, as, e.g., depicted in Fig. 5(a) and (b). Redundancy constraints related to the control include, e.g., the connection of multiple clutches in a row or a direct connection of a brake to the wheels. Further, a certain component instance may only be used if a lower component instance is already present. This can mathematically be defined as, $v_{\lambda, \tau, \iota+1} \geq v_{\lambda, \tau, \iota}$. If, we consider, e.g., a gear pair, then the second gear pair may only be introduced if the first gear pair is present.

3) Physical Constraints: The physical constraints prevent concepts to be generated that can not be constructed from a mechanical point of view. Rotational directions of shafts should be respected to guarantee adequate functioning of the system. A counter example of the rotational direction mismatch can be observed in Fig. 5(a) and (b). Furthermore, co-axiallity of shafts is essential to build a construction. Co-axiallity in this context refers to the difference in center lines between two shafts. A counter example is illustrated in Fig. 5(c) and (d). Dimensioning and packaging could also be included as physical constraints, yet is not part of this research.

4) Application Specific Constraints: The components in Table II are provided with layout types (series, parallel). In addition, the following application specific constraints are related to a powertrain equipped with a conventional front-transverse CVT system:

1) the centres of the output shaft of the ICE and differential should not be constructed in a series layout;

2) the differential should be connected to a gear pair to reduce the torque inside the transmission; and, finally,

3) a brake should not be connected to the engine, since it is the only input of the transmission in this case study.

As a result of the $\mathrm{CP}$ algorithm, many advanced and novel CVT topologies are generated at the end of step (2), i.e., 465923 in 5.5 hours of computation time ${ }^{1}$ reducing the design space with an order of magnitude difference of $O(22)$; and, as example three different types: (a) shaft-to-shaft (SS) single-range (SR), (b) powersplit (PS), (c) mixed (MI) CVT, are shown in Fig. 6, respectively.

\section{Topology CVT Evaluation, Step (3)}

The evaluation is performed in four sequential steps, as indicated on the left side of Fig. 3 and are discussed below.

1) Topology Screening, Step (3a): The concept of topologies being equivalent in structure except for their labeling is referred to as being isomorphic [27]. Two topologies $T_{\lambda, i}$ and $T_{\lambda, j}$ are isomorphic, if there is a bijection $\mathfrak{b}$ such that $\mathfrak{b}\left(V_{\lambda}\left(T_{\lambda, i}\right)\right)=V_{\lambda}\left(T_{\lambda, j}\right), \mathfrak{b}^{-1}\left(V_{\lambda}\left(T_{\lambda, j}\right)\right)=V_{\lambda}\left(T_{\lambda, i}\right)$ and $\mathfrak{b}\left(E_{\lambda}\left(T_{\lambda, i}\right)\right)=E_{\lambda}\left(T_{\lambda, j}\right)$. In order to assure topology uniqueness, similar topologies that are defined as being equal in structure and component type, yet different in component

\footnotetext{
${ }^{1}$ Intel Core 17 CPU @ 2.80 GHz; 8.00 GB RAM
}

instant labelling, will be removed using a hash function. This function computes the sum of the component types (based on unique vertex numbering) over each powerflow path from input to the output for all feasible topologies. Accordingly, similar hash functions values are used as a measure to create an unique set of feasible topologies. In addition, the connectivity of the graph is also verified by checking if the vertices from the input to output during the different transmission operation modes are connected. The design space is after this step (3a) (programmed in Matlab) further reduced to 2525 feasible topologies, which is an order of magnitude difference of $O(2)$ compared to step (2).

2) Mode Searching, Step (3b): Mode searching here is defined as the search process to the attainable driving modes of a transmission. Changing operation mode requires disengaging one set of clutches/brakes and engaging another set, as specified by a discrete control schedule, which is the key result of this evaluation step. Additionally, all feasible planetary gear set arrangements are obtained, hereby, reducing the number of design alternatives. In [12] and [13], we provide the detailed analysis of determining the functional-operational modes for arbitrary topologies with an arbitrary amount of clutches, brakes and planetary gears for a full-electric or hybrid powertrain design equipped with one combustion engine and/or maximum two electric machines. Based on the component types $\tau$ and instances $\iota$ as defined in the library, the plant and control design variables as function of time $t$, are specified as follows, respectively: $v_{3}^{p}=\left[v_{3,2,1}^{p}, v_{3,2,2}^{p}, v_{3,2,3}^{p}, v_{3,3,1}^{p}\right], v_{3}^{c}(t)=$ $\left[v_{3,1,1}^{c}(t), v_{3,4,1}^{c}(t), v_{3,4,2}^{c}(t), v_{3,5,1}^{c}(t)\right]$. Where the gear speed ratios $v_{3,2,1 \ldots 3}^{p}$, the PGS ratio $v_{3,3,1}^{p}$ and the variable variator speed ratio $v_{3,1,1}^{c}(t)$ are bounded by an upper and lower bound, i.e., $v_{3, \tau, \iota}^{p} \in\left[\underline{v}_{3, \tau, \iota}^{p}, \bar{v}_{3, \tau, \iota}^{p}\right] \subset \mathbb{R}, v_{3,1,1}^{c}(t) \in\left[\underline{v}_{3,1,1}^{c}, \bar{v}_{3,1,1}^{c}\right] \subset \mathbb{R}$; and, the clutch and brake controlled states are binary variables $\left\{v_{3,4,1}^{c}, v_{3,4,2}^{c}, v_{3,5,1}^{c}\right\} \subseteq\{0,1\}^{3} \subset \mathbb{N}^{3}$, i.e., zero means disengaged and one means engaged. For this evaluation step, a physical system model is automatically generated using Simscape and an example for the mixed CVT type is shown in the Fig 7(a). In Fig. 7(b), an exhaustive search over the control design variables $v_{3}^{c}$ given $v_{3}^{p, 0}=[3,0.5,2,2]$ as the nominal set of plant design parameters for the overall CVT speed ratio (vertical axis), i.e., simulating all possible combinations (horizontal axis) of the clutch, brake states and for simplicity a finite number of variator speed ratios $v_{3,1,1}^{c}$ at the bounds (under-, overdrive) and an intermediate (medium) value are depicted. This topology contains two clutches, resulting in four possible control combinations for each fixed chosen variator ratio with a total of 12 possible combinations. Since, the physical network modelling approach is hardware-based, infeasible clutch combinations (i.e., 'control design variable combination' number 4, 8 and 12) do not result in a feasible overall CVT ratio, however, provides a simulation error. This occurs, e.g., if all clutches are engaged simultaneously. Two operation modes for forward and reverse driving in a 'Shaft-to-Shaft' (Cf. Fig. 6(d), CL1 open and CL2 closed) and 'Powersplit' mode (CL1 closed and CL2 open); and, one 'Idle' driving mode (both clutches open) can be observed in Fig. 7(b) that are based on $12-3=9$ admissible-controlled states. Finally, the mode search algorithm automatically derives a (discrete) control schedule, containing the clutch states, $v_{3,4,1,2}^{c}$, for each operation mode, $M \in\{$ 'Idle', 'Shaft-to-Shaft', 'Powersplit' $\}:=\{1,2,3\}$. 


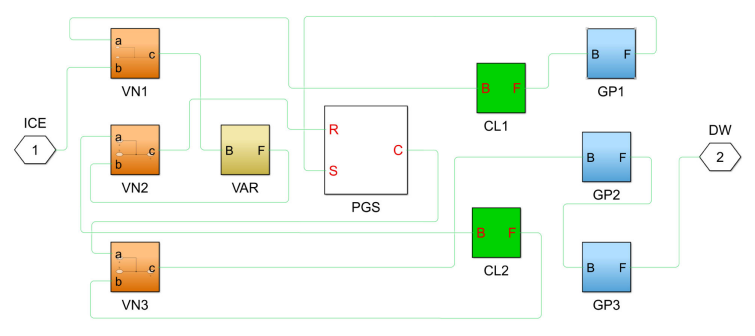

(a)

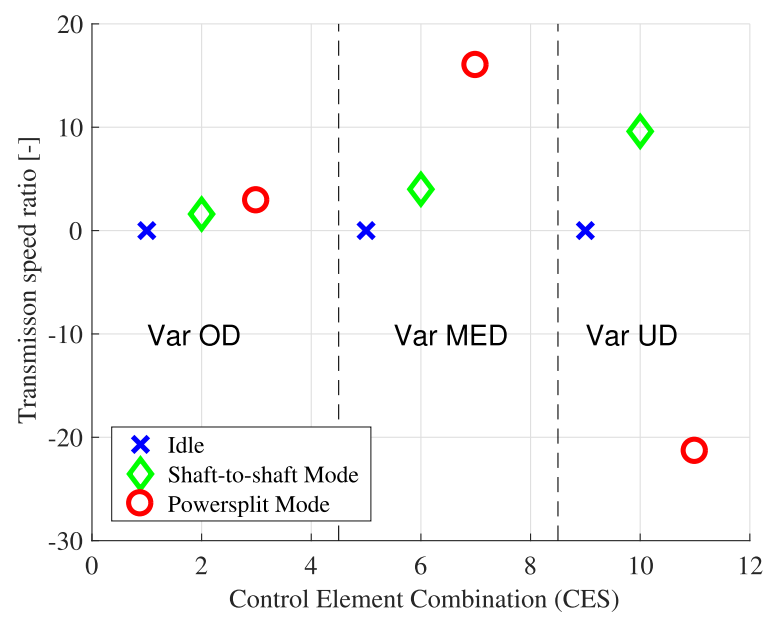

(b)

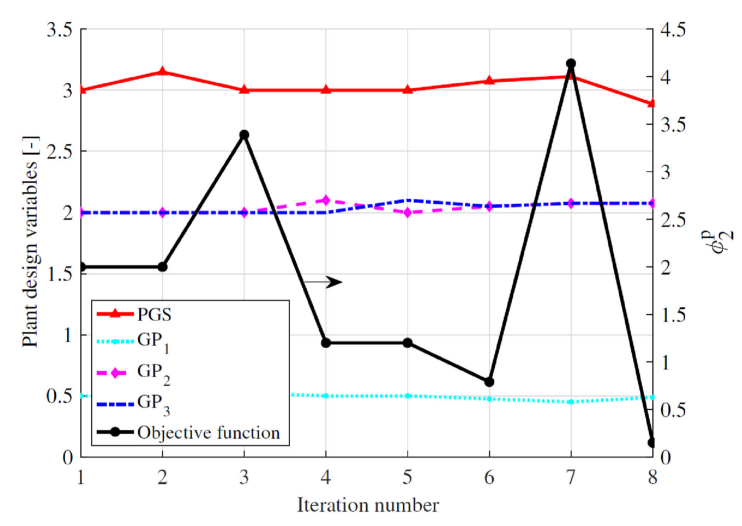

(c)

Fig. 7. Step ( $3 b$ and $3 c)$, mode searching and performance evaluation for the mixed CVT topology (Cf. Fig. 6(d)). (a) Example of an automatic generated Simscape model for a mixed CVT topology (Cf. Fig. 6(d)) used for the subsequent evaluation steps (3b) and (3c). (b) Global search for the overall CVT speed ratio, $v_{2,2,1}^{p}\left(v_{3}^{c}(M), v_{3}^{p}\right)$ over the admissible-controlled states $(12-3=9$ feasible combinations) for three fixed variator speed ratios: underdrive (UD): $\bar{v}_{3,1,1}^{c}=2.4$; medium (MED): $v_{3,1,1}^{c}=1$; overdrive (OD): $\underline{v}_{3,1,1}^{c}=0.4$, respectively. (c) Minimization of criterion $\phi_{2}^{p}$ (vertical axis - right side) by variation of the plant design variables $v_{3}^{p}$ (vertical axis - left side) as a function of the iteration step for driving mode $M=3$. The initial plant design variables are chosen as: $v_{3}^{p, 0}=[3,0.5,2,2]$ for this diagram.

3) Performance Evaluation, Step (3c): In this case study, the performance evaluation step will further filter out infeasible designs using plant design specifications $\phi_{\lambda}^{p}($.$) alone. The max-$ imum overall CVT speed ratio value at system level $(\lambda=2)$, denoted as $\bar{v}_{2,3,1}^{p}$, can be specified for the following vehicle requirements, e.g., acceleration time, hill climb from standstill, vehicle creep, etc. It should be noted that the system design specification can be extended to fuel usage $(1 / \mathrm{km})$ minimization on a driving cycle as targets $\phi_{\lambda}^{c}($.$) or constraints g_{\lambda}^{c}($.$) from$ higher to lower system levels in the ATC framework. Due to the coupling between the control and plant design, the co-design method will then be fully exploited, yet is seen as future work. Here, for reasons of space, we focus on a key quasi-static performance requirement as example: hill climbing in a Powerplit mode $M=3$ with the variator speed constant in medium (MED), or $v_{3,1,1}^{c}(t)=1$ resulting in the minimization of a local (coupling) objective $\phi_{2}^{p}($.$) defined as the (absolute) differ-$ ence between a specified maximum CVT speed ratio $\bar{v}_{2,3,1}^{p}$ and the response value from the CVT plant design analysis model, $v_{2,3,1}^{p}($.$) :$

$$
\begin{aligned}
& \min _{v_{3}^{p}} \phi_{2}^{p}=\left|\bar{v}_{2,3,1}^{p}-v_{2,3,1}^{p}\left(v_{3}^{c}(M), v_{3}^{p}\right)\right| \\
\text { s.t. : } & g_{3}^{p}\left(v_{3,2 \ldots 3,1 \ldots 3}^{p}\right): v_{3,2 \ldots 3,1 \ldots 3}^{p}-\bar{v}_{3,2 \ldots 3,1 \ldots 3}^{p} \leq 0 ; \\
& \underline{v}_{3,2 \ldots 3,1 \ldots 3}^{p}-v_{3,2 \ldots 3,1 \ldots 3}^{p} \leq 0
\end{aligned}
$$

The criterion $\phi_{2}^{p}$ over $v_{3}^{p}$ for given $\bar{v}_{2,3,1}^{p}=18(-)$ and $v_{3}^{c}(3)=[1 ; 1 ; 0]$ is minimized in eight iterations, as shown in Fig. 7(c). The influence of the remaining admissible driving modes on the plant design specifications are also automatically and efficiently analysed; and, used to determine the optimal set of plant parameters for the defined vehicle requirements in step. The topologies unable to meet the defined design specifications are filtered out reducing the design space with an order of magnitude difference of $O(1)$ to finally 635 feasible topologies. These 635 feasible topologies can be categorized as will be described next. Note that the total computation time of step (3) (including classification) takes approximately 20 hours.

4) Topology Classification, Step (3d): The generated feasible topologies (635) can be categorized with respect to their mode operations [28]: (i) shaft-to-shaft (SS) (or nonpowersplit); (ii) powersplit (PS) (consisting of one or more PGSs); and, (iii) mixed (MI) types ((i) and (ii) combined). The later two types (ii) and (iii) can also be further classified with input-coupled or output-coupled CVT types. This type of classification is different compared to [5], since the classification is based on the hybrid powertrain typologies and components in [5]. Opposed to a (classical) single-range (SR) CVT with a dual-range (DR) CVT, the ratio range of a CVT can be used twice [24], which may results in significantly reducing the CVT dimensions, while maintaining the same ratio coverage. Note that in this case study, the generated topologies consist of only a single planetary gear set. Furthermore, note that,

i) the shaft-to-shaft (SS) topologies contain only a single path for the power to flow [28], [29]. The total transmission ratio spread of a shaft-to-shaft single-range (SS) topology, sometimes referred to as a conventional (standard) topology, is formed in the variator alone. An example of a SS topology is depicted in Fig. 6(b).

ii) the power paths for the powersplit (PS) topologies are divided into two branches: one with a fixed ratio and another with the variable ratio. Both paths rejoin by a torque coupler (output-coupled) or planetary gear set (inputcoupled, e.g., as depicted in Fig. 6(c)); 


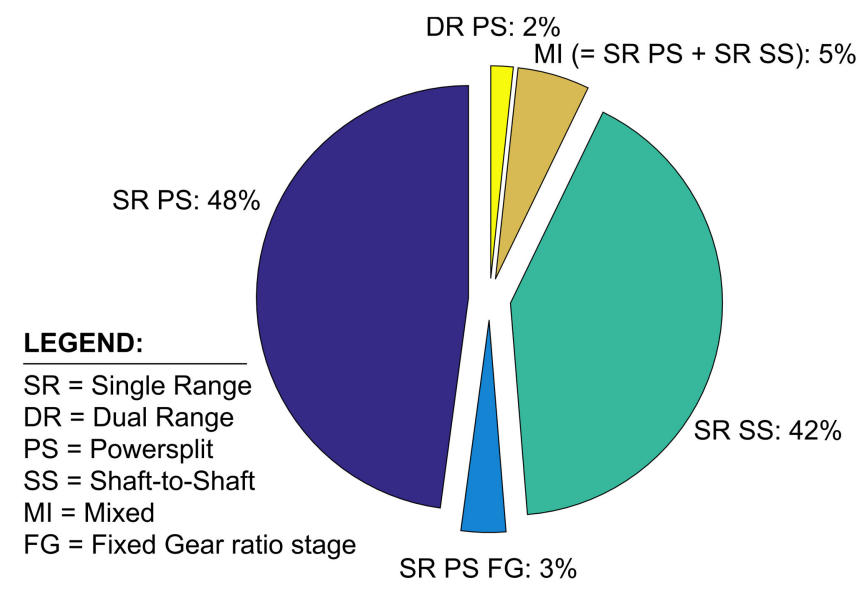

Fig. 8. Step (3d), topology classification: the relative distribution of results of the categorized feasible CVT topologies (in total 635).

iii) the mixed (MI) topology types refer to the shiftable combination of the two before mentioned classes [28] and a novel generated example is depicted in Fig. 6(d).

On top of these three CVT topology classes, a fixed-gear (FG) ratio-stage can be added, e.g., to increase the transmission efficiency by decoupling the variator in overdrive ratio [24]. Range shifting can be performed by a clutch-to-clutch shift and can be executed simultaneously with a variator shift [30]. The distribution of the categorized CVT topologies $\{\mathrm{SR}, \mathrm{DR}\} \times$ $\{\mathrm{SS}, \mathrm{PS}, \mathrm{MI}\} \times\{\mathrm{FG}\}$ (with a total of 635 feasible topologies) using the CDS algorithm are shown in Fig. 8: three SR classes (93\%); one DR (2\%); and, one MI (5\%) class. The SR class can be futher categorized into: PS (48\%); PS FG (3\%); and, SS type $(42 \%)$. The largest amount $(93 \%)$ of the feasible topologies are of the single-range (SR) type.

5) Guidance, Step (4): In this case study, the feasible CVT topologies are synthesized using subsystems for the conventional and parallel (P2 type) hybrid configuration (Cf. Fig. 1, $\lambda=2$ ). Therefore, as a guidance step from a higher to a lower system level, the synthesized topologies require having a single input and output shaft as new design consistency constraints at $\lambda=2$. In a similar manner, the CVT subsystems at $\lambda=2$ consist of subsubsystems at $\lambda=3$ (clutches, brakes) that allows the transmission to switch between operation modes. Accordingly, the mode operations and speed ratios as controllable states require having feasible physical actuator hardware from lower system levels at $\lambda=5$. Thereby, sufficient fast, smooth and efficient (on demand) operation as design specifications create additional design consistency constraints in a subsequent guidance step to lower levels from $\lambda=3$ to $\lambda=5$. As discussed in Section III, in future work, the focuss will be on new mechanical- and (electrical-) hydraulic actuation system topologies (Cf. Fig. 2) for the variator, clutches and brakes.

\section{E. Computation Time of Step (3)}

The time spent for a single simulation run on the automated topology evaluation is in detail analysed in Fig. 9 for the mixed CVT topology (Cf. Fig. 6(d)). The exploded parts indicate the

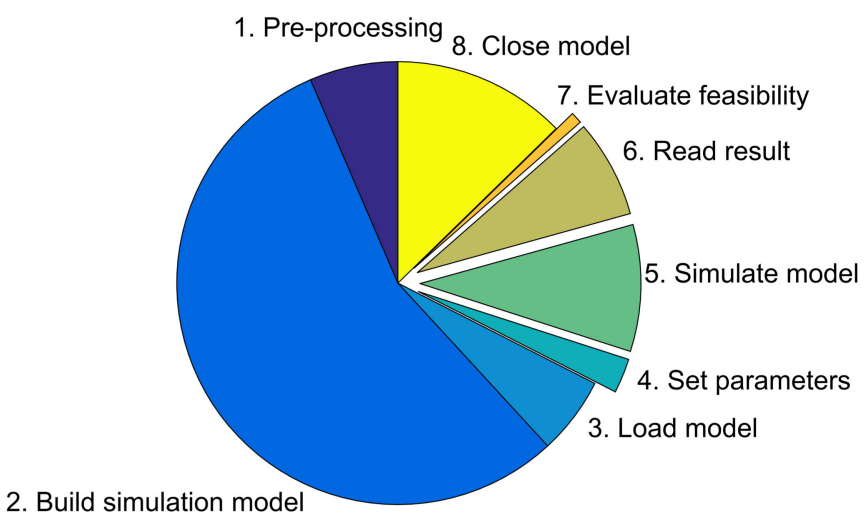

Fig. 9. Step (3), computation time of the simulation-based evaluation phase: the pie chart shows the relative time distribution from 1. 'Pre-processing' towards an automatic generated SimScape model in 2, which is used for the subsequent simulation-based evaluation steps (3. - 7.) towards the last step 8 . 'Close model'. For this example, the total simulation time including only one simulation run equals $2.4 \mathrm{~s}$ (complete pie chart) for the mixed CVT topology (Cf. Fig. 6(d)). Searching for all operation modes and evaluating the performances require additionally 12 runs for mode searching and 8 runs for performance evaluation (by repeating steps 4. - 7.) 1 run (already incl. in the pie-chart)) $\times$ $20 \%$ (steps $4 .-7$.) of the $2.4 \mathrm{~s}$ equals in total $11.5 \mathrm{~s}$.

phases that are repeated multiple times. For example, the mixed CVT topology containing one variator, which is divided into three fixed operation ratios (UD, MED, OD) and two clutches results in 12 simulation model (Cf. 7(b), Step (3b)) and 8 performance evaluation (Cf. 7(c), Step (3c)) run repeats (step 2. 7.) for each PGS arrangement.

The total time spent for one topology evaluation, including one simulation run, equals 2.4 seconds $^{2}$ and including the repeated additional simulation model and evaluation runs in total $11.5 \mathrm{~s}^{3}$. No structural changes are made to the simulation model during performing a global search, i.e., varying the design variables. To increase the computational speed, simulations are performed using pre-compiled simulation models. In this way, simulation tasks are rapidly performed without regenerating C-code. In Simscape, this is implemented by means of 'fast restart'. During the first simulation run, the model is compiled, initialized, ran and saved. For the next simulation run, the model is already initialized; hence, the compilation time for the next simulations is avoided. Further, it can be observed that the building the simulation model takes more than half of the total simulation time $(1.4 \mathrm{~s})$.

\section{CONCLUSION}

A framework for CDS from higher towards lower system levels has been developed. Inherently, moving from a higher system level to a lower system level and using a powertrain and a high-tech CVT design case study this required the development of a new set of constraint types; automated simulation-based evaluation algorithms; and, new requirements on the guidance

\footnotetext{
${ }^{2}$ Intel Core i7 CPU @ 2.80 GHz; 8.00 GB RAM

${ }^{3}$ For the average feasible CVT topology, the total simulation and evaluation time takes approximately $18 \mathrm{~s}$ resulting with six PGS arrangments and 2525 feasible topologies (step (3a)) an overal computation time (devided by four parallel processes on a quad-core CPU) of $75 / 4 \approx 19$ hours, which is in the order of the measured time of 20 hours (see step (3c)).
} 
steps towards lower system levels. In addition, seen as a new technical contribution, many novel and innovative CVT system topologies designs (635) were generated in a relative fast and efficient manner for a chosen AWD hybrid-powertrain topology. The proposed framework contributes by representing the plant design problem as a topology and component design problem over multiple system levels. This results in a nested graph model, whereby from top to bottom system levels, the process of design synthesis is modelled as a cyclic process of representation, generation, evaluation and guidance steps and can be combined with multidisciplinary optimization methods as analytical targets cascading including control design in future work.

\section{REFERENCES}

[1] E. Silvas, T. Hofman, N. Murgovski, L. Etman, and M. Steinbuch, "Review of optimization strategies for system-level design in hybrid electric vehicles," IEEE Trans. Veh. Technol., vol. 66, no. 1, pp. 57-70, Jan. 2017.

[2] E. Silvas, E. Bergshoeff, T. Hofman, and M. Steinbuch, "Comparison of bi-level optimization frameworks for sizing and control of a hybrid electric vehicle," in Proc. IEEE Veh. Power Propulsion Conf., Oct. 2014, pp. 1-6.

[3] J. Allison and D. Herber, "Multidisciplinary design optimization of dynamic engineering systems," AIAA J., vol. 52, no. 4, pp. 691-710, 2014.

[4] A. Sangiovanni-Vincentelli, "Quo Vadis SLD: Reasoning about trends and challenges of system-level design," Proc. IEEE, vol. 95, no. 3, pp. 467506, Mar. 2007.

[5] E. Silvas, T. Hofman, A. Serebrenik, and M. Steinbuch, "Functional and cost-based automatic generator for hybrid vehicles topologies," IEEE/ASME Trans. Mechatronics, vol. 20, no. 4, pp. 1561-1572, Aug. 2015.

[6] P. Nuzzo et al., "A contract-based methodology for aircraft electric power system design," IEEE Access, vol. 2, pp. 1-25, 2014.

[7] G. Barbieri, "Platform-based design: Methodology refinement and application to cyber-physical production systems," Ph.D. dissertation, Dept. Sci. Methods Eng., Univ. Modena Reggio Emilia, Modena, Italy, 2016.

[8] J. Wan, A. Canedo, and M. A. Faruque, "Functional model-based design methodology for automotive cyber-physical systems," IEEE Syst. J., vol. 11, no. 4, pp. 2028-2039, Dec. 2017.

[9] B. Helms, K. Shea, and F. Hoisl, "A framework for computational design synthesis based on graph-grammars and function-behavior-structure," ASME. Int. Design Eng. Tech. Conf. Comput. Inf. Eng. Conf., Volume 8: 14th Design Manufacturing Life Cycle Conf.; 6th Symp. Int. Design Design Education; 21st Int. Conf. Design Theory Methodology, Parts A and B, pp. 841-851, doi: 10.1115/DETC2009-86851.

[10] J. Cagan, M. Campbell, S. Finger, and T. Tomiyama, "A framework for computational design synthesis: Models and applications," J. Comput. Inf. Sci. Eng., vol. 5, pp. 171-181, 2005.

[11] A. Kort, J. Wijkniet, T. Hofman, and A. Serebrenik, "Automated topology synthesis for transmission actuation systems," to be published, 2018.

[12] W. van Harselaar, T. Hofman, and M. Brouwer, "Automated dynamic modeling of arbitrary hybrid and electric drivetrain topologies," IEEE Trans. Veh. Technol., vol. 67, no. 8, pp. 6921-6934, Aug. 2018.

[13] W. van Harselaar, T. Hofman, and M. Brouwer, "A generic transmission model for hybrid electric drives," in Proc. Int. VDI Congr. Drivetrain Veh., 2018.

[14] S. Delprat, T. Hofman, and S. Paganelli, "Hybrid vehicle energy management: Singular optimal control," IEEE Trans. Veh. Technol., vol. 66, no. 11, pp. 9654-9666, Nov. 2017.

[15] J. Sowa, Laws, Facts, and Contexts: Foundations for Multimodal Reasoning. Dordrecht, The Netherlands: Springer, 2003, pp. 145-184.

[16] S. Eppinger and T. Browning, Design Structure Matrix Methods and Applications. Cambridge, MA, USA: MIT Press, 2012.

[17] C. Gorbea, "Vehicle architecture and lifecycle cost analysis in a new age of architectural competition," Ph.D. dissertation, Inst. Product Develop., Technische Universität München, München, Germany, 2011.
[18] C. Münzer, "Constraint-based methods for automated computational design synthesis of solution spaces," Ph.D. dissertation, Inst. Product Develop., ETH Zürich, Zürich, Switzerland, 2015.

[19] "SWI Prolog." [Online]. Available: http://www.swi-prolog.org/download/ stable

[20] MathWorks, "Model and simulate multi-domain physical systems." [Online]. Available: https://nl.mathworks.com/products/simscape.html

[21] H. Kim et al., "Target cascading in vehicle redesign: A class VI truck study," Int. J. Veh. Des., vol. 29, no. 3, pp. 199-225, 2002.

[22] J. Oguri, H. Itou, and N. Akiyame, "Enhanced performance of next generation LINEARTRONIC (SUBARU)," VDI Wissensforum, pp. 1-13, 2016.

[23] D. Foster, "A new torque converter CVT fit for moving market requirements," in Proc. 12th Int. CTI Symp., MI, USA, May 2018, pp. 1-12.

[24] A. Englisch, A. Teubert, B. Walter, K. Braun, S. Penner, and M. Jost, "CVT-The transmission concept of the future," in Proc. Schaeffler Symp., 2014, pp. 139-151.

[25] Punch Powertrain, "Multi-mode hybrid system, HS2," 2018. [Online]. Available: http://www.punchpowertrain.com/nl/producten/17/hs2

[26] Punch Powertrain, "Electric powertrain, EP1," 2018. [Online]. Available: http://www.punchpowertrain.com/n1/producten/12/ep1

[27] L. Tsai, Mechanism Design: Enumeration of Kinematic Structures According to Function (Mechanical and Aerospace Engineering Series). Boca Raton, FL, USA: CRC Press, 2000.

[28] P. Linares, V. Méndez, and H. Catalán, "Design parameters for continuously variable power-split transmissions using planetaries with three active shafts," J. Terramech., vol. 47, no. 5, pp. 323-335, 2010.

[29] J. Kress, "Hydrostatic power-splitting transmissions for wheeled vehicles: Classification and theory of operation," 1968, SAE Tech. Paper 680549.

[30] F. van der Sluis, H. Lamers, and G. J. van Spijk, "The two-stage push-belt CVT," in Proc. Global Powertrain Congr., 2003, vol. 25, pp. 16-25.

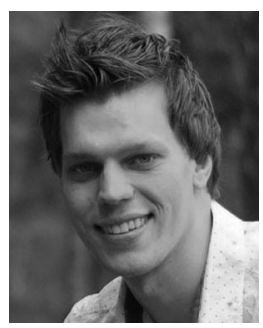

Jan Wijkniet was born in Lienden, The Netherlands, in 1990. He received the M.Sc. (Hons.) degree in automotive technology from Eindhoven University of Technology, Eindhoven, The Netherlands, in 2016. Since 2016, he has been a Systems Engineer with the Advanced Development Department, Punch Powertrain N.V., Eindhoven, The Netherlands. His research interests include concept generation and model-based evaluation of complex dynamical systems. His main focus is on advanced vehicular powertrain applications with automated transmissions in particular.

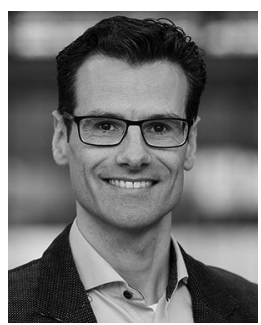

Theo Hofman was born in Utrecht, The Netherlands, in 1976. He received the M.Sc. (Hons.) and the $\mathrm{Ph}$.D. degrees from Eindhoven University of Technology, Eindhoven, The Netherlands, in 1999 and 2007, respectively, both in mechanical engineering. From 1999 to 2003, he was a Researcher and the Project Manager with the R\&D Department, Thales. Cryogenics B.V., Eindhoven, The Netherlands. From 2003 to 2007, he was a Scientific Researcher with the Drivetrain Innovations B.V., Eindhoven, The Netherlands. Since 2010, he has been an Assistant Professor with the Control Systems Technology group. His research interests include system design optimization methods for complex dynamical engineering systems and automated computational design synthesis for discrete topology design. 